\title{
New 4-methylated and 19-oxygenated steroids from the Formosan soft coral Nephthea erecta
}

\author{
Shi-Yie Cheng ${ }^{a}$, Chang-Feng Dai ${ }^{b}$, Chang-Yih Duh ${ }^{a, c, *}$ \\ a Department of Marine Biotechnology and Resources, National Sun Yat-sen University, Kaohsiung, Taiwan \\ ${ }^{\mathrm{b}}$ Institute of Oceanography, National Taiwan University, Taipei, Taiwan \\ c Center of Asia-Pacific Marine Researches, National Sun Yat-sen University, Kaohsiung, Taiwan
}

\section{A R T I C L E I N F O}

Article history:

Received 2 March 2007

Received in revised form

11 April 2007

Accepted 9 May 2007

Published on line 17 May 2007

\begin{abstract}
A B S T R A C T
Two new 4-methylated steroids, erectasteroids A and B (1 and 2), six new 19-oxygenated steroids, erectasteroids C-H (3-8) and two known 19-oxygenated steroids (9 and 10) were isolated from the acetone solubles of the Formosan soft coral Nephthea erecta. The structures were elucidated by extensive NMR spectroscopic analysis and their cytotoxicity against selected cancer cells was measured in vitro.
\end{abstract}

(c) 2007 Elsevier Inc. All rights reserved.

\section{Keywords:}

Nephthea erecta

Cytotoxicity

\section{Introduction}

Soft corals of the genus Nephthea are rich in terpenoids and steroids [1-14]. As part of our search for bioactive substances from marine organisms, the Formosan soft coral Nephthea erecta Kükenthal (Nephtheidae) was studied because the acetone extracts showed significant cytotoxicity to HT-29 (human colon adenocarcinoma) and P-388 (mouse lymphocytic leukemia) cell cultures as determined by standard procedures $[15,16]$. Bioassay-guided fractionation resulted in the isolation of two new cytotoxic 4-methylated steroids, erectasteroids A and B (1 and 2), six new 19-oxygenated steroids, erectasteroids $\mathrm{C}-\mathrm{H}$ (3-8), and two known 19-oxygenated steroids (9 and 10) [19].

\section{Experimental}

\subsection{General}

Optical rotations were determined on a JASCO P1020 polarimeter. UV spectra were obtained on a Hitachi U-3210 spectrophotometer, and IR spectra were recorded on a JASCO FT/IR-4100 spectrophotometer. NMR spectra were recorded on a Varian Inova 500 or a Bruker Avance 300 spectrometer. Chemical shifts are given in $\delta$ (ppm) and coupling constants in Hz. ESIMS were recorded by ESI FT-MS on a BRUKER APEX II mass spectrometer. Si gel 60 (Merck, 230-400 mesh) was used for column chromatography; precoated Si gel plates (Merck, Kieselgel $60 \mathrm{~F}_{254}, 0.25 \mathrm{~mm}$ ) were used for TLC analysis.

\footnotetext{
* Corresponding author at: Department of Marine Biotechnology and Resources, National Sun Yat-sen University, Kaohsiung, Taiwan. Tel.: +886 7 5252000x5036; fax: +886 75255020 .

E-mail address: yihduh@mail.nsysu.edu.tw (C.-Y. Duh). 0039-128X/\$ - see front matter @ 2007 Elsevier Inc. All rights reserved. doi:10.1016/j.steroids.2007.05.001
} 


\subsection{Animal material}

The soft coral N. erecta was collected at Green Island, off Taiwan, in September 2005, at a depth of 3-4 m and was stored for 4 weeks in a freezer until extraction. A voucher specimen, NSUGN-080, was deposited in the Department of Marine Biotechnology and Resources, National Sun Yat-sen University, Taiwan.

\subsection{Extraction and isolation}

The bodies of the soft coral N. erecta were freeze dried to give $1.8 \mathrm{~kg}$ of a solid, which was extracted with acetone $(20 \mathrm{~L} \times 3)$. The acetone solubles was evaporated to give a dark brown residue $(35.0 \mathrm{~g})$, which was chromatographed on a silica gel column using eluents of increasing polarity from $n$-hexane to EtOAc to obtain fractions 1-27. Fraction 11 was subjected to RP-18 HPLC column chromatography $\left(95 \% \mathrm{MeOH}\right.$ in $\left.\mathrm{H}_{2} \mathrm{O}\right)$ to afford compounds 1 (6 mg) and 2 (1 mg). Compounds 3 (5 mg), 4 (4 mg), 5 ( $5 \mathrm{mg})$, and 6 (1 mg) were obtained from fraction 20 by RP-18 HPLC column chromatography $\left(90 \% \mathrm{MeOH}\right.$ in $\left.\mathrm{H}_{2} \mathrm{O}\right)$. Repeated chromatography of fraction 22 over RP-18 HPLC column ( $84 \% \mathrm{MeOH}$ in $\mathrm{H}_{2} \mathrm{O}$ ) led to the isolation of compounds 7 (1 mg), 8 (3 mg), 9 (4 mg), and 10 (7 mg).

\subsubsection{Erectasteroid A (1)}

Colorless syrup. $[\alpha]_{\mathrm{D}}^{24}+3.2\left(\mathrm{c} 0.6, \mathrm{CHCl}_{3}\right)$; $\mathrm{UV}(\mathrm{MeOH}) \lambda \max (\log \varepsilon)$ : 221 (3.63) nm; IR (KBr) v $v_{\max }$ : 3337, 2926, 1682, 1634, 1437, 1385, 1239, 1031, 921, $708 \mathrm{~cm}^{-1} ;{ }^{1} \mathrm{H}$ NMR, see Table $1 ;{ }^{13} \mathrm{C}$ NMR, see Table 2; HRESIMS, $m / z 451.3555$ (calcd. for $\mathrm{C}_{29} \mathrm{H}_{48} \mathrm{O}_{2} \mathrm{Na}$, 451.3552).

\subsubsection{Erectasteroid B (2)}

Colorless syrup. $[\alpha]_{\mathrm{D}}^{24}-62.0$ (c $0.1, \mathrm{CHCl}_{3}$ ); UV (MeOH) $\lambda_{\max }$ $(\log \varepsilon)$ : 222 (3.62) nm; IR (KBr) v $\nu_{\max }$ : 3343, 2925, 1707, 1686, 1638, 1557, 1454, 1381, 1036, 927, $736 \mathrm{~cm}^{-1} ;{ }^{1} \mathrm{H} \mathrm{NMR}$, see Table $1 ;{ }^{13} \mathrm{C}$ NMR, see Table 2; HRESIMS, $\mathrm{m} / \mathrm{z} 465.3346$ (calcd. for $\mathrm{C}_{29} \mathrm{H}_{46} \mathrm{O}_{3} \mathrm{Na}$, 465.3344).

\subsubsection{Erectasteroid C (3)}

Limpid molasses. $[\alpha]_{\mathrm{D}}^{24}+13.6$ (c 0.5, $\left.\mathrm{CHCl}_{3}\right)$; IR (KBr) v $v_{\max }$ : 3390, 2952, 1734, 1457, 1369, 1239, 1046, 1020, 968, $739 \mathrm{~cm}^{-1} ;{ }^{1} \mathrm{H}$ NMR, see Table $3 ;{ }^{13} \mathrm{C}$ NMR, see Table 2; HRESIMS, $\mathrm{m} / \mathrm{z} 493.3292$ (calcd. for $\mathrm{C}_{30} \mathrm{H}_{46} \mathrm{O}_{4} \mathrm{Na}$, 493.3294).

\subsubsection{Erectasteroid D (4)}

Limpid molasses. $[\alpha]_{\mathrm{D}}^{24}+29.0$ (c 0.4, $\mathrm{CHCl}_{3}$ ); IR ( $\left.\mathrm{KBr}\right) v_{\max }: 3405$, $2948,1723,1671,1463,1374,1254,1046,754 \mathrm{~cm}^{-1} ;{ }^{1} \mathrm{H}$ NMR, see Table 3; ${ }^{13} \mathrm{C}$ NMR, see Table 2; HRESIMS, $\mathrm{m} / \mathrm{z} 481.3292$ (calcd. for $\mathrm{C}_{29} \mathrm{H}_{46} \mathrm{O}_{4} \mathrm{Na}$, 481.3294).

\subsubsection{Erectasteroid E (5)}

Limpid molasses. $[\alpha]_{D}^{24}+9.6$ (c 0.5, $\mathrm{CHCl}_{3}$ ); UV (MeOH) $\lambda_{\max }$ $(\log \varepsilon): 231$ (3.67) nm; IR (KBr) $v_{\max }: 3405,2943,1734,1458,1369$, 1244, 1046, 1020, $739 \mathrm{~cm}^{-1} ;{ }^{1} \mathrm{H}$ NMR, see Table $3 ;{ }^{13} \mathrm{C}$ NMR, see Table 2; HRESIMS, $m / z 483.3453$ (calcd. for $\mathrm{C}_{29} \mathrm{H}_{48} \mathrm{O}_{4} \mathrm{Na}$, 483.3450).

Table $1-{ }^{1} \mathrm{H}$ NMR spectral data ${ }^{\mathrm{a}}(500 \mathrm{MHz})$ of 1 and 2 in $\mathrm{CDCl}_{3}$

2

\begin{tabular}{|c|c|c|}
\hline $1 \alpha$ & $1.02 \mathrm{~m}$ & $1.32 \mathrm{~m}$ \\
\hline $1 \beta$ & $1.72 \mathrm{~m}$ & $2.05 \mathrm{~m}$ \\
\hline $2 \alpha$ & $1.81 \mathrm{~m}$ & $2.31 \mathrm{ddd}(15.0,6.5,3.5)$ \\
\hline $2 \beta$ & $1.48 \mathrm{~m}$ & $2.49 \operatorname{td}(15.0,6.5)$ \\
\hline 3 & $3.08 \operatorname{td}(10.0,5.0)^{b}$ & \\
\hline 4 & $1.29 \mathrm{~m}$ & $2.35 \mathrm{~m}$ \\
\hline 5 & $0.72 \mathrm{~m}$ & $1.18 \mathrm{~m}$ \\
\hline $6 \alpha$ & $1.28 \mathrm{~m}$ & $0.92 \mathrm{~m}$ \\
\hline $6 \beta$ & $1.52 \mathrm{~m}$ & $1.53 \mathrm{~m}$ \\
\hline $7 \alpha$ & $0.78 \mathrm{~m}$ & $1.24 \mathrm{~m}$ \\
\hline $7 \beta$ & $1.69 \mathrm{~m}$ & $1.68 \mathrm{~m}$ \\
\hline 8 & $1.30 \mathrm{~m}$ & \\
\hline 9 & $0.61 \operatorname{td}(11.5,4.3)$ & $0.60 \mathrm{~m}$ \\
\hline $11 \alpha$ & $1.80 \mathrm{~m}$ & $1.03 \mathrm{~m}$ \\
\hline $11 \beta$ & $1.29 \mathrm{~m}$ & $1.55 \mathrm{~m}$ \\
\hline $12 \alpha$ & $1.12 \mathrm{~m}$ & $1.18 \mathrm{~m}$ \\
\hline $12 \beta$ & $1.97 \mathrm{dt}(12.5,3.0)$ & $2.06 \mathrm{~m}$ \\
\hline 14 & $0.98 \mathrm{~m}$ & $1.25 \mathrm{~m}$ \\
\hline $15 \alpha$ & $1.58 \mathrm{~m}$ & $1.02 \mathrm{~m}$ \\
\hline $15 \beta$ & $1.00 \mathrm{~m}$ & $0.86 \mathrm{~m}$ \\
\hline $16 \alpha$ & $1.66 \mathrm{~m}$ & $1.86 \mathrm{~m}$ \\
\hline $16 \beta$ & $1.06 \mathrm{~m}$ & $1.36 \mathrm{~m}$ \\
\hline 17 & $1.15 \mathrm{~m}$ & $1.12 \mathrm{~m}$ \\
\hline 18 & $0.70 \mathrm{~s}$ & $0.99 \mathrm{~s}$ \\
\hline 19 & $0.83 \mathrm{~s}$ & $1.23 \mathrm{~s}$ \\
\hline 20 & $2.03 \mathrm{~m}$ & $2.04 \mathrm{~m}$ \\
\hline 21 & $0.89 d(6.5)$ & $0.88 d(6.5)$ \\
\hline 22 & $\begin{array}{l}2.68 \mathrm{dd}(15.5,3.0) \\
2.40 \mathrm{dd}(15.5,10.0)\end{array}$ & $\begin{array}{l}2.67 \text { dd }(15.5,5.3) \\
2.40 \mathrm{dd}(15.5,10.0)\end{array}$ \\
\hline 25 & 2.92 heptet (7.0) & 2.92 heptet (6.5) \\
\hline 26 & $1.01 \mathrm{~d}(7.0)$ & $1.10 \mathrm{~d}(6.5)$ \\
\hline 27 & $1.03 \mathrm{~d}(7.0)$ & $1.03 \mathrm{~d}(6.5)$ \\
\hline 28 & $5.91 \mathrm{~s}, 5.67 \mathrm{~s}$ & $5.91 \mathrm{~s}, 5.67 \mathrm{~s}$ \\
\hline 29 & $0.95 \mathrm{~d}(7.0)$ & $1.00 \mathrm{~d}(6.0)$ \\
\hline
\end{tabular}

a Assigned by COSY, HSQC, NOESY, and HMBC experiments.

${ }^{\mathrm{b}} \mathrm{J}$ values (in $\mathrm{Hz}$ ) in parentheses.

\subsubsection{Erectasteroid $F(6)$}

Limpid molasses. $[\alpha]_{\mathrm{D}}^{24}-70.0$ (c 0.1, $\mathrm{CHCl}_{3}$ ); IR (KBr) v $v_{\max }$ : 3380, 2932, 1738, 1452, 1374, 1233, 1036, 890, $739 \mathrm{~cm}^{-1} ;{ }^{1} \mathrm{H}$ NMR, see Table 3; ${ }^{13} \mathrm{C}$ NMR, see Table 2; HRESIMS, $\mathrm{m} / \mathrm{z} 495.3477$ (calcd. for $\mathrm{C}_{30} \mathrm{H}_{48} \mathrm{O}_{4} \mathrm{Na}$, 495.3450).

\subsubsection{Erectasteroid $F(7)$}

Limpid molasses. $[\alpha]_{\mathrm{D}}^{24}-44.0$ (c $0.1, \mathrm{CHCl}_{3}$ ); IR (KBr) $v_{\max }$ : 3318, 2948, 1677, 1640, 1525, 1447, 1385, 1187, 1031, 927, 734, $634 \mathrm{~cm}^{-1} ;{ }^{1} \mathrm{H}$ NMR, see Table $4 ;{ }^{13} \mathrm{C}$ NMR, see Table 2; HRESIMS, $\mathrm{m} / \mathrm{z} 453.3345$ (calcd. for $\mathrm{C}_{28} \mathrm{H}_{46} \mathrm{O}_{3} \mathrm{Na}, 453.3344$ ).

\subsubsection{Erectasteroid $H(8)$}

White amorphous powder. $[\alpha]_{\mathrm{D}}^{24}-16.0$ (c $0.3, \mathrm{CHCl}_{3}$ ); UV $(\mathrm{MeOH}) \lambda_{\max }(\log \varepsilon): 232(3.65) \mathrm{nm}$; IR $(\mathrm{KBr}) v_{\max }$ : 3333, 2937, 1651, 1458, 1380, 1031, 962, $884 \mathrm{~cm}^{-1} ;{ }^{1} \mathrm{H}$ NMR, see Table 4; ${ }^{13} \mathrm{C}$ NMR, see Table 2; HRESIMS, $\mathrm{m} / \mathrm{z} 451.3186$ (calcd. for $\mathrm{C}_{28} \mathrm{H}_{44} \mathrm{O}_{3} \mathrm{Na}$, 451.3188).

\subsection{Cytotoxicity testing}

P-388 cells were kindly supplied by Prof. J.M. Pezzuto, formerly of the Department of Medicinal Chemistry and Pharmacog- 


\begin{tabular}{|c|c|c|c|c|c|c|c|c|c|c|}
\hline C & $1^{\mathrm{a}}$ & $2^{a}$ & $3^{b}$ & $4^{b}$ & $5^{b}$ & $6^{a}$ & $7^{c}$ & $8^{d}$ & $9^{c}$ & $10^{\mathrm{d}}$ \\
\hline 1 & 36.8 & 39.8 & 33.2 & 33.2 & 33.2 & 34.0 & 34.5 & 33.1 & 34.5 & 33.1 \\
\hline 2 & 31.0 & 39.4 & 28.4 & 31.7 & 31.7 & 31.5 & 32.4 & 25.9 & 32.8 & 25.9 \\
\hline 3 & 76.6 & 213.7 & 70.9 & 70.9 & 70.9 & 71.2 & 72.1 & 70.7 & 72.2 & 70.7 \\
\hline 4 & 39.2 & 44.6 & 41.6 & 41.2 & 41.6 & 42.1 & 43.1 & 41.4 & 42.6 & 41.4 \\
\hline 5 & 50.9 & 53.6 & 140.0 & 140.0 & 140.0 & 141.1 & 142.5 & 138.7 & 140.2 & 138.7 \\
\hline 6 & 21.1 & 18.5 & 126.7 & 126.9 & 126.7 & 127.7 & 128.6 & 129.7 & 131.2 & 129.7 \\
\hline 7 & 32.2 & 37.5 & 75.1 & 75.1 & 75.1 & 64.9 & 65.9 & 72.0 & 73.5 & 72.0 \\
\hline 8 & 34.8 & 73.5 & 37.8 & 37.8 & 37.8 & 38.7 & 40.1 & 41.1 & 42.5 & 41.0 \\
\hline 9 & 54.5 & 55.8 & 48.5 & 48.5 & 48.4 & 42.5 & 44.0 & 49.2 & 50.7 & 49.2 \\
\hline 10 & 36.0 & 36.6 & 41.4 & 41.6 & 41.4 & 40.3 & 43.1 & 41.1 & 43.3 & 41.0 \\
\hline 11 & 28.5 & 21.3 & 21.7 & 21.7 & 21.6 & 21.4 & 22.6 & 21.6 & 23.1 & 21.6 \\
\hline 12 & 40.0 & 40.8 & 39.7 & 39.6 & 39.6 & 39.3 & 41.1 & 40.0 & 41.5 & 40.2 \\
\hline 13 & 42.7 & 43.2 & 43.0 & 42.9 & 43.1 & 42.3 & 43.5 & 42.9 & 44.3 & 42.9 \\
\hline 14 & 56.5 & 59.1 & 56.5 & 56.6 & 56.5 & 50.2 & 51.7 & 57.2 & 58.8 & 57.2 \\
\hline 15 & 24.1 & 18.9 & 23.8 & 25.0 & 25.0 & 24.2 & 25.0 & 28.4 & 27.4 & 23.6 \\
\hline 16 & 24.1 & 27.8 & 24.9 & 28.8 & 28.6 & 28.2 & 29.4 & 31.3 & 30.2 & 28.3 \\
\hline 17 & 56.6 & 57.0 & 55.4 & 55.2 & 55.2 & 55.6 & 57.2 & 55.6 & 57.0 & 55.7 \\
\hline 18 & 12.1 & 13.5 & 12.1 & 12.3 & 12.4 & 11.7 & 12.4 & 11.6 & 13.0 & 11.3 \\
\hline 19 & 13.3 & 13.0 & 62.8 & 62.9 & 62.9 & 64.7 & 63.6 & 62.1 & 63.6 & 62.1 \\
\hline 20 & 33.5 & 32.8 & 35.7 & 39.6 & 40.2 & 35.7 & 37.0 & 40.4 & 41.7 & 35.8 \\
\hline 21 & 19.6 & 19.4 & 18.7 & 20.9 & 20.5 & 18.7 & 19.2 & 19.9 & 21.7 & 18.0 \\
\hline 22 & 45.3 & 45.3 & 36.1 & 137.9 & 135.8 & 34.6 & 36.0 & 135.8 & 139.7 & 36.1 \\
\hline 23 & 202.9 & 202.6 & 31.7 & 126.4 & 129.3 & 30.8 & 32.0 & 129.2 & 127.6 & 31.3 \\
\hline 24 & 156.0 & 155.9 & 39.4 & 41.9 & 153.1 & 156.8 & 157.8 & 153.1 & 42.9 & 39.4 \\
\hline 25 & 27.8 & 27.8 & 28.0 & 28.5 & 29.3 & 33.8 & 34.9 & 29.3 & 29.9 & 27.8 \\
\hline 26 & 21.9 & 21.9 & 22.5 & 22.2 & 22.4 & 21.8 & 22.3 & 21.5 & 22.9 & 21.8 \\
\hline 27 & 22.0 & 22.0 & 22.8 & 22.3 & 22.0 & 22.0 & 22.4 & 21.2 & 22.8 & 21.6 \\
\hline 28 & 120.7 & 120.8 & & & 109.7 & 106.0 & 106.8 & 108.8 & & \\
\hline 29 & 15.1 & 11.6 & & & & & & & & \\
\hline \multirow{2}{*}{ OAc } & & & 171.4 & 171.4 & 171.4 & 170.6 & & & & \\
\hline & & & 21.7 & 21.7 & 21.6 & 21.0 & & & & \\
\hline
\end{tabular}

\footnotetext{
a Spectrum recorded at $125 \mathrm{MHz}$ in $\mathrm{CDCl}_{3}$.

b Spectrum recorded at $75 \mathrm{MHz}$ in $\mathrm{CDCl}_{3}$.

c Spectrum recorded at $125 \mathrm{MHz}$ in $\mathrm{CD}_{3} \mathrm{OD}$. The values are in ppm downfield from TMS and assignments were made by DEPT, COSY, HMQC, and $\mathrm{HMBC}$ experiments.

d Spectrum recorded at $75 \mathrm{MHz}$ in $\mathrm{CD}_{3} \mathrm{OD}$.
}

nosy, University of Illinois at Chicago; HT-29 was purchased from the American Type Culture Collection. Cytotoxic assays were carried out according to the procedure described previously $[15,16]$.

\section{Results and discussion}

Compound 1 had a molecular formula of $\mathrm{C}_{29} \mathrm{H}_{48} \mathrm{O}_{2}$ as established by HRESIMS and NMR spectroscopic data. The ${ }^{1} \mathrm{H}$ NMR spectrum revealed the presence of two tertiary methyls $\left(\delta_{\mathrm{H}}\right.$ 0.70 and 0.83$)$, four secondary methyls $\left(\delta_{\mathrm{H}} 0.95,0.89,1.01\right.$, and 1.03), and an oxymethine $\left[\delta_{\mathrm{H}} 3.08(1 \mathrm{H}, \mathrm{td}, J=10.0,5.0 \mathrm{~Hz})\right]$. The presence of a vinylic methylene conjugated to a carbonyl group was revealed by NMR signals [ $\left(\delta_{\mathrm{H}} 5.91\right.$ and 5.67$) ; \delta_{\mathrm{C}}$ $120.7\left(\mathrm{CH}_{2}\right), 156.0(\mathrm{qC})$, and 202.9 (qC)] (Tables 1 and 2), as well as from IR absorption at 1682 and $1634 \mathrm{~cm}^{-1} \cdot{ }^{13} \mathrm{C}$ NMR and DEPT spectra of 1 also exhibited nine $\mathrm{sp}^{3}$ methylenes, nine $\mathrm{sp}^{3}$ methines, and two $\mathrm{sp}^{3}$ quaternary carbons. The NMR and HRMS data could account for 2 of the 6 degrees of unsaturation, suggesting the tetracyclic nature of 1 . Twentynine carbons including six methyls and comparison of NMR

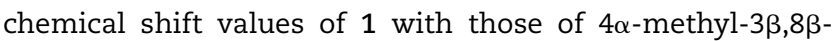
dihydroxy-5 $\alpha$-ergost-24(28)-en-23-one reported from the soft coral Litophyton viridis suggested that 1 may be a 8 -deoxy analogue of $4 \alpha$-methyl-3 $\beta, 8 \beta$-dihydroxy- $5 \alpha$-ergost-24(28)-en23-one [17]. Interpretation of the ${ }^{1} \mathrm{H}-{ }^{1} \mathrm{H}$ COSY spectrum led to partial structures I and II (Fig. 1). Partial structures I and II were connected through the conjugated enone by HMBC correlations between $\mathrm{H}_{3}-26, \mathrm{H}_{3}-27 / \mathrm{C}-24, \mathrm{C}-25$, between $\mathrm{H}_{2}-28 / \mathrm{C}-23$, $\mathrm{C}-24, \mathrm{C}-25$, and between $\mathrm{H}_{2}-22 / \mathrm{C}-23$. Rings $\mathrm{A}$ and $\mathrm{B}$ were elucidated on the basis of HMBC cross-peaks between $\mathrm{H}_{3}-19 / \mathrm{C}-1$, $\mathrm{C}-5, \mathrm{C}-9, \mathrm{C}-10$ and $\mathrm{H}-4 / \mathrm{C}-29$, whereas rings $\mathrm{C}$ and D were completed based on HMBC correlations between $\mathrm{H}_{3}-18 / \mathrm{C}-12, \mathrm{C}-13$, C-14, C-17. The NOESY correlations (Fig. 2) observed between $\mathrm{H}-11 \beta$ and $\mathrm{H}-8, \mathrm{H}-11 \beta$ and $\mathrm{H}_{3}-18, \mathrm{H}-11 \beta$ and $\mathrm{H}_{3}-19, \mathrm{H}-9$ and $\mathrm{H}-14, \mathrm{H}_{3}-18$ and $\mathrm{H}-8, \mathrm{H}_{3}-19$ and $\mathrm{H}-8, \mathrm{H}_{3}-18$ and $\mathrm{H}-20, \mathrm{H}-3$ and $\mathrm{H}-1 \alpha, \mathrm{H}-3$ and $\mathrm{H}-5$, and $\mathrm{H}_{3}-19$ and $\mathrm{H}-4 \beta$ in 1 confirmed the relative configurations for each ring junction and chiral center.

Compound 2 had a molecular formula of $\mathrm{C}_{29} \mathrm{H}_{46} \mathrm{O}_{3}$ as determined by HRESIMS. The spectral data (Tables 1 and 2) resembled those of 1 except for NMR signals due to a ketone at C-3 ( $\delta_{C}$ 213.7) and a tertiary hydroxyl at C$8\left(\delta_{C}\right.$ 73.5). Comparison of NMR chemical shift values of 
Table $3-{ }^{1} \mathrm{H}$ NMR spectral data ${ }^{\mathrm{a}}$ of 3-5 (300 MHz), and $6(500 \mathrm{MHz})$ in $\mathrm{CDCl}_{3}$

\begin{tabular}{|c|c|c|c|c|}
\hline & 3 & 4 & 5 & 6 \\
\hline $1 \alpha$ & $1.06 \mathrm{~m}$ & $1.08 \mathrm{~m}$ & $1.11 \mathrm{~m}$ & $1.14 \mathrm{~m}$ \\
\hline $1 \beta$ & $1.92 \mathrm{~m}$ & $1.97 \mathrm{~m}$ & $1.97 \mathrm{~m}$ & $2.06 \mathrm{~m}$ \\
\hline $2 \alpha$ & $1.84 \mathrm{~m}$ & $1.85 \mathrm{~m}$ & $1.86 \mathrm{~m}$ & $1.90 \mathrm{~m}$ \\
\hline $2 \beta$ & $1.38 \mathrm{~m}$ & $1.43 \mathrm{~m}$ & $1.39 \mathrm{~m}$ & $1.43 \mathrm{~m}$ \\
\hline 3 & $3.60 \mathrm{~m}$ & $3.60 \mathrm{~m}$ & $3.61 \mathrm{~m}$ & 3.63 dddd $(10.0,8.3,5.5,4.5)$ \\
\hline $4 \alpha$ & $2.38 \mathrm{~m}$ & $2.39 \mathrm{~m}$ & $2.40 \mathrm{dd}(13.2,2.7)$ & $2.40 \mathrm{~m}$ \\
\hline $4 \beta$ & $2.21 \mathrm{~m}$ & $2.21 \mathrm{~m}$ & $2.24 \mathrm{~m}$ & $2.34 \mathrm{~m}$ \\
\hline 6 & $5.58 \mathrm{br} \mathrm{s}$ & $5.58 \mathrm{br} \mathrm{s}$ & $5.58 \mathrm{br} \mathrm{s}$ & $5.86 \mathrm{~d}(4.5)$ \\
\hline 7 & 4.96 br d $(8.6)^{b}$ & $4.96 \mathrm{br} d(8.5)$ & 4.96 br d (8.7) & $3.91 \mathrm{dd}(4.5,4.0)$ \\
\hline 8 & $2.14 \mathrm{~m}$ & $2.18 \mathrm{~m}$ & $2.17 \mathrm{~m}$ & $1.78 \operatorname{td}(12.0,4.0)$ \\
\hline 9 & $1.08 \mathrm{~m}$ & $1.08 \mathrm{~m}$ & $1.06 \mathrm{~m}$ & $1.28 \mathrm{~m}$ \\
\hline $11 \alpha$ & $1.66 \mathrm{~m}$ & $1.67 \mathrm{~m}$ & $1.70 \mathrm{~m}$ & $1.62 \mathrm{~m}$ \\
\hline $11 \beta$ & $1.58 \mathrm{~m}$ & $1.60 \mathrm{~m}$ & $1.64 \mathrm{~m}$ & $1.54 \mathrm{~m}$ \\
\hline $12 \alpha$ & $1.27 \mathrm{~m}$ & $1.12 \mathrm{~m}$ & $1.19 \mathrm{~m}$ & $1.18 \mathrm{~m}$ \\
\hline $12 \beta$ & $2.07 \mathrm{~m}$ & $2.02 \mathrm{~m}$ & $2.06 \mathrm{~m}$ & $2.04 \mathrm{~m}$ \\
\hline 14 & $1.02 \mathrm{~m}$ & $1.07 \mathrm{~m}$ & $1.08 \mathrm{~m}$ & $1.36 \mathrm{~m}$ \\
\hline $15 \alpha$ & $1.31 \mathrm{~m}$ & $1.38 \mathrm{~m}$ & $1.42 \mathrm{~m}$ & $1.71 \mathrm{~m}$ \\
\hline $15 \beta$ & $1.09 \mathrm{~m}$ & $1.25 \mathrm{~m}$ & $1.30 \mathrm{~m}$ & $1.14 \mathrm{~m}$ \\
\hline $16 \alpha$ & $1.40 \mathrm{~m}$ & $1.60 \mathrm{~m}$ & $1.66 \mathrm{~m}$ & $1.93 \mathrm{~m}$ \\
\hline $16 \beta$ & $1.26 \mathrm{~m}$ & $1.25 \mathrm{~m}$ & $1.27 \mathrm{~m}$ & $1.31 \mathrm{~m}$ \\
\hline 17 & $1.03 \mathrm{~m}$ & $1.07 \mathrm{~m}$ & $1.17 \mathrm{~m}$ & $1.22 \mathrm{~m}$ \\
\hline 18 & $0.74 \mathrm{~s}$ & $0.76 \mathrm{~s}$ & $0.78 \mathrm{~s}$ & $0.71 \mathrm{~s}$ \\
\hline 19 & $3.89 \mathrm{~d}$ (11.5), $3.66 \mathrm{~d}(11.5)$ & $3.88 \mathrm{~d}(11.5), 3.65 \mathrm{~d}(11.5)$ & $3.89 \mathrm{~d}(11.5), 3.66 \mathrm{~d}(11.5)$ & $4.53 \mathrm{~d}(12.0), 3.97 \mathrm{~d}(12.0)$ \\
\hline 20 & $1.34 \mathrm{~m}$ & $2.02 \mathrm{~m}$ & $2.14 \mathrm{~m}$ & $1.44 \mathrm{~m}$ \\
\hline 21 & $0.91 \mathrm{~d}(6.4)$ & $1.00 \mathrm{~d}(6.5)$ & $1.05 \mathrm{~d}(6.8)$ & $0.96 \mathrm{~d}(7.0)$ \\
\hline 22 & $1.03 \mathrm{~m}$ & $5.21 \mathrm{dd}(15.0,7.8)$ & $5.58 \mathrm{dd}(15.8,8.8)$ & $1.54 \mathrm{~m}, 1.18 \mathrm{~m}$ \\
\hline 23 & $1.23 \mathrm{~m}, 1.79 \mathrm{~m}$ & $5.23 \mathrm{dt}(15.0,7.8)$ & $5.93 \mathrm{~d}(15.8)$ & $2.10 \mathrm{~m}$ \\
\hline 24 & $1.13 \mathrm{~m}$ & $1.81 \mathrm{~m}$ & & \\
\hline 25 & $1.50 \mathrm{~m}$ & $1.56 \mathrm{~m}$ & 2.54 heptet (6.8) & 2.23 heptet $(7.0)$ \\
\hline 26 & $0.86 \mathrm{~d}(6.6)$ & $0.85 d(6.1)$ & $1.07 \mathrm{~d}(6.8)$ & $1.03 \mathrm{~d}(7.0)$ \\
\hline 27 & $0.86 \mathrm{~d}(6.6)$ & $0.85 d(6.1)$ & $1.05 d(6.8)$ & $1.03 \mathrm{~d}(7.0)$ \\
\hline 28 & & & $4.82 \mathrm{~s}, 4.84 \mathrm{~s}$ & $4.72 \mathrm{~s}, 4.66 \mathrm{~s}$ \\
\hline OAc & $2.03 \mathrm{~s}$ & $2.02 \mathrm{~s}$ & $2.01 \mathrm{~s}$ & $2.03 \mathrm{~s}$ \\
\hline
\end{tabular}

a Assigned by COSY, HSQC, NOESY, and HMBC experiments.

${ }^{\mathrm{b}} \mathrm{J}$ values (in $\mathrm{Hz}$ ) in parentheses.

2 with those of Jone's oxidation products of $4 \alpha$-methyl$3 \beta, 8 \beta$-dihydroxy-5 $\alpha$-ergost-24(28)-en-23-one suggested that 2 may be $4 \alpha$-methyl-8 $\beta$-hydro-xy- $5 \alpha$-ergost-24(28)-en-3,23dione [17]. HMBC correlations (Fig. 3) from $\mathrm{H}_{2}-2 / \mathrm{H}-4 / \mathrm{H}_{3}-29$ to $\mathrm{C}-3$ and from $\mathrm{H}-7 / \mathrm{H}-9 / \mathrm{H}-14$ to $\mathrm{C}-8$ helped positioning the ketone and the tertiary hydroxyl at C-3 and C-8, respectively. ${ }^{13} \mathrm{C}$ NMR spectral data of 2 were not reported and ${ }^{1} \mathrm{H}$ spectral data were not completely assigned in the literature [17].

Compound 3 had a molecular formula of $\mathrm{C}_{29} \mathrm{H}_{48} \mathrm{O}_{4}$ as determined by HRESIMS. The IR spectrum showed the presence of hydroxyl group(s) $\left(3390 \mathrm{~cm}^{-1}\right)$ and an ester group $\left(1734 \mathrm{~cm}^{-1}\right)$. The presence of two oxymethines and one oxymethylene was shown by the ${ }^{1} \mathrm{H}$ NMR $\left[\delta_{\mathrm{H}} 3.60(1 \mathrm{H}, \mathrm{m}), 4.96(1 \mathrm{H}, \mathrm{br} \mathrm{d}, J=8.6 \mathrm{~Hz})\right.$, $3.89(1 \mathrm{H}, \mathrm{d}, J=11.5 \mathrm{~Hz}), 3.66(1 \mathrm{H}, \mathrm{d}, J=11.5 \mathrm{~Hz})]$ and ${ }^{13} \mathrm{C} \mathrm{NMR}$ [ $\left.\delta_{\mathrm{C}} 70.9(\mathrm{CH}), 75.1(\mathrm{CH}), 62.8\left(\mathrm{CH}_{2}\right)\right]$ spectra. The ${ }^{1} \mathrm{H}$ and ${ }^{13} \mathrm{C}$ NMR spectra also showed signals due to four methyl groups [ $\left.\delta_{\mathrm{H}} 0.74(3 \mathrm{H}, \mathrm{s}), 0.86(6 \mathrm{H}, \mathrm{d}, J=6.6 \mathrm{~Hz}), 0.91(3 \mathrm{H}, \mathrm{d}, J=6.4 \mathrm{~Hz})\right]$, and a trisubstituted double bond $\left[\delta_{\mathrm{H}} 5.58(1 \mathrm{H}, \mathrm{br} \mathrm{s}), \delta_{\mathrm{C}} 126.7\right.$ (CH), 140.0 (qC)]. The ${ }^{13} \mathrm{C}$ NMR and DEPT spectra of 3 also exhibited $10 \mathrm{sp}^{3}$ methylenes, $5 \mathrm{sp}^{3}$ methines, and $2 \mathrm{sp}^{3}$ quaternary carbons. This evidence suggested that 3 contained the same steroid nucleus (A, B, C, and D rings) as of 24methylenecholest-5-en-3 $\beta, 7 \beta, 19$-triol 7-acetate [18] except for the side chain. Comparison of the ${ }^{13} \mathrm{C}$ NMR chemical shifts with those of cholesterol indicate unambiguously that compound 3 is cholest-5-en-3 $\beta, 7 \beta, 19$-triol 7-acetate. The COSY, HMBC (Fig. 4), and NOESY spectra (Fig. 5) of 3 confirmed this, showing the expected correlations.

The spectral data of 4 and 5 were analogous to those of 3, except for NMR signals due to the side chain. As shown in Tables 2 and 3, signals due to a 22-E disubstituted double bond $\left[\delta_{\mathrm{H}} 5.21(1 \mathrm{H}, \mathrm{dd}, J=15.0,7.8 \mathrm{~Hz})\right.$ and $5.23(1 \mathrm{H}, \mathrm{dt}, J=15.0,7.8 \mathrm{~Hz})$; $\delta_{\mathrm{C}} 137.9(\mathrm{CH})$ and $\left.126.4(\mathrm{CH})\right]$ were observed in 4 , and those due to a 22E,24(28)-diene $\left[\delta_{\mathrm{H}} 5.58(1 \mathrm{H}, \mathrm{dd}, J=15.8,8.8 \mathrm{~Hz})\right.$ and 5.93 $(1 \mathrm{H}, \mathrm{d}, J=15.8 \mathrm{~Hz}) ; 4.82(1 \mathrm{H}, \mathrm{s})$ and $4.84(1 \mathrm{H}, \mathrm{s}) ; \delta_{\mathrm{C}} 135.8(\mathrm{CH})$, $129.3(\mathrm{CH}), 153.1(\mathrm{qC})$, and $\left.109.7\left(\mathrm{CH}_{2}\right)\right]$ appeared in the side chain of 5 .

Compound 6 had a molecular formula of $\mathrm{C}_{30} \mathrm{H}_{48} \mathrm{O}_{4}$ as determined by HRESIMS. The spectral data (Tables 2 and 3) showed some similarity to those of 24-methylenecholesta-5en-3 $\beta, 7 \beta, 19$-triol [18] except for the 19-hydroxyl was replaced by an 19-acetoxyl [ $\delta_{\mathrm{H}} 4.53$ and $3.97(2 \mathrm{H}$, each $d, J=12.0 \mathrm{~Hz})$ and $2.03(3 \mathrm{H}, \mathrm{s}) ; \delta_{\mathrm{C}} 64.9\left(\mathrm{CH}_{2}\right), 21.0\left(\mathrm{CH}_{3}\right)$, and $\left.170.6(\mathrm{qC})\right]$ in 6. COSY correlations from $\mathrm{H}-20$ to $\mathrm{H}_{2}-22$ and from $\mathrm{H}_{2}-22$ to $\mathrm{H}_{2}$ 23 as well as HMBC correlations from $\mathrm{H}_{2}-19$ to C-5/C-10/C-1 and from $\mathrm{H}_{2}-28$ to $\mathrm{C}-23$ helped ascertain this assignment. The 

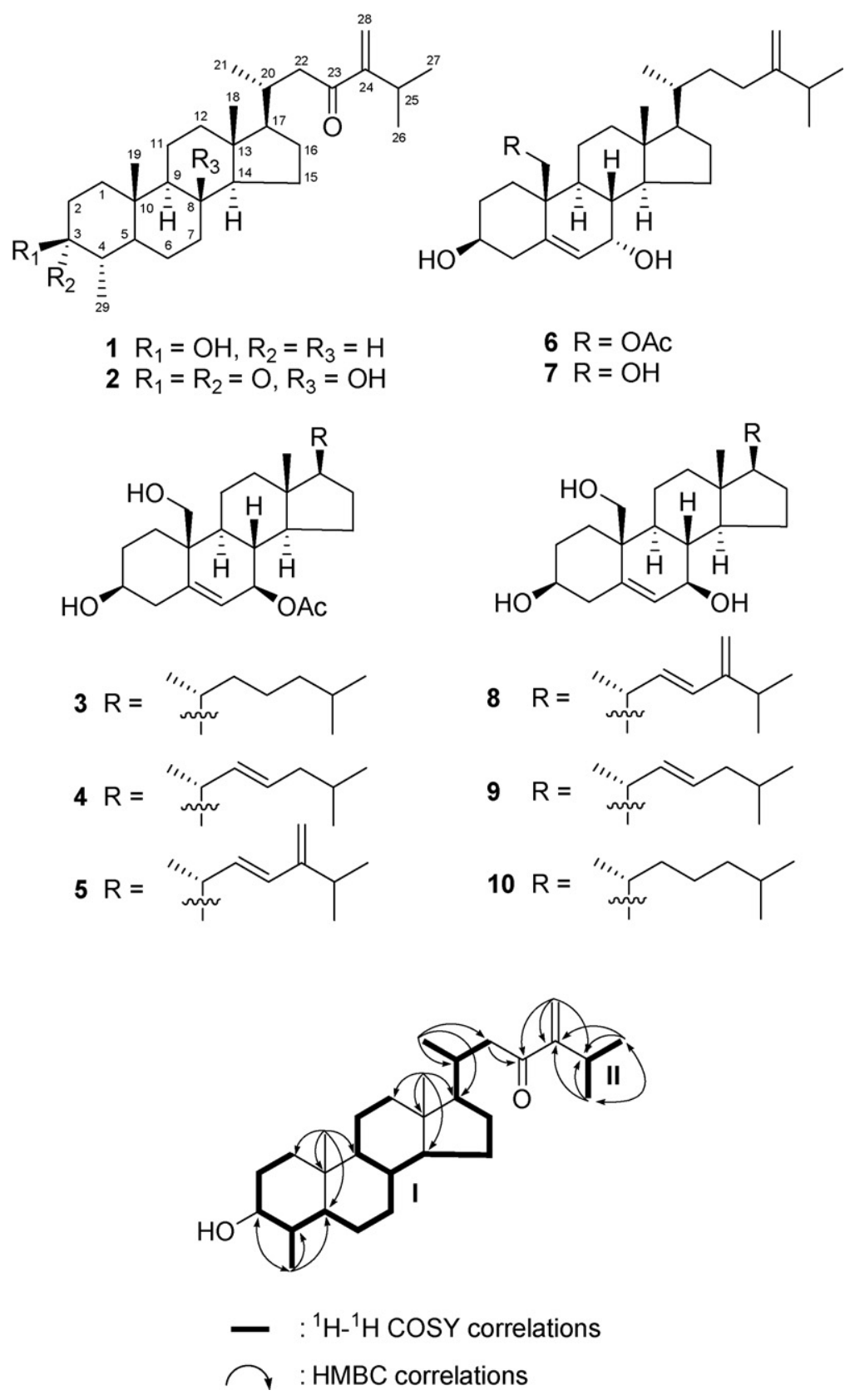

Fig. $1-{ }^{1} \mathrm{H}-{ }^{1} \mathrm{H}$ COSY and key HMBC correlations of 1.

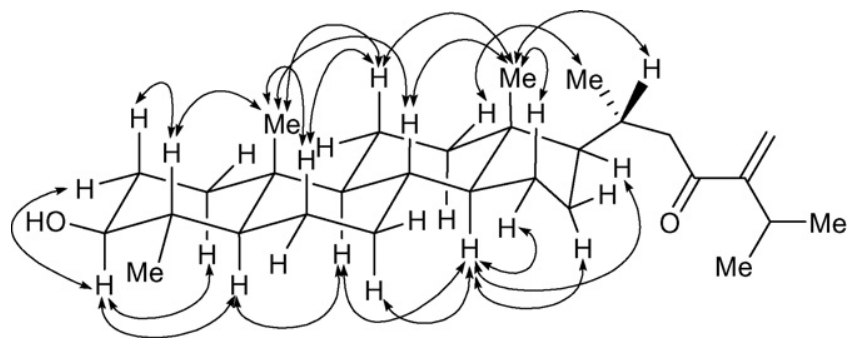

Fig. 2 - Selected NOESY correlations of 1.

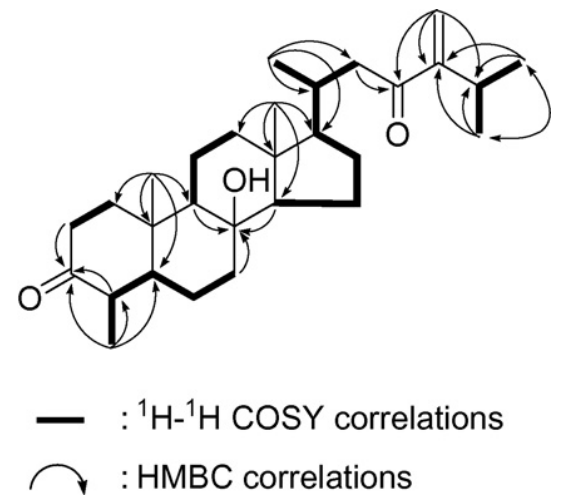

Fig. $3-{ }^{1} \mathrm{H}-{ }^{1} \mathrm{H}$ COSY and key HMBC correlations of 2. 
Table $4-{ }^{1} \mathrm{H}$ NMR spectral data ${ }^{\mathrm{a}}$ of 7, $9(500 \mathrm{MHz})$, and 8, $10(500 \mathrm{MHz})$ in $\mathrm{CD}_{3}$ OD

7

\begin{tabular}{|c|c|c|c|c|}
\hline $1 \alpha$ & $1.11 \mathrm{~m}$ & $0.98 \mathrm{~m}$ & $0.98 \mathrm{~m}$ & $0.99 \mathrm{~m}$ \\
\hline $1 \beta$ & $1.96 \mathrm{~m}$ & $2.09 \mathrm{~m}$ & $2.05 \mathrm{~m}$ & $2.08 \mathrm{~m}$ \\
\hline $2 \alpha$ & $1.77 \mathrm{~m}$ & $1.81 \mathrm{~m}$ & $1.78 \mathrm{~m}$ & $1.84 \mathrm{~m}$ \\
\hline $2 \beta$ & $1.42 \mathrm{~m}$ & $1.45 \mathrm{~m}$ & $1.40 \mathrm{~m}$ & $1.44 \mathrm{~m}$ \\
\hline 3 & $3.53 \mathrm{~m}$ & $3.47 \mathrm{~m}$ & $3.46 \mathrm{~m}$ & $3.45 \mathrm{~m}$ \\
\hline $4 \alpha$ & $2.33 \mathrm{~m}$ & $2.30 \mathrm{~m}$ & $2.31 \mathrm{~m}$ & $2.31 \mathrm{~m}$ \\
\hline $4 \beta$ & $2.30 \mathrm{~m}$ & $2.24 \mathrm{~m}$ & $2.25 \mathrm{~m}$ & $2.28 \mathrm{~m}$ \\
\hline 6 & $5.79 \mathrm{~d}(4.5)^{\mathrm{b}}$ & 5.49 br s & 5.49 br s & 5.49 br s \\
\hline 7 & $3.79 \mathrm{t}(4.5)$ & $3.67 \mathrm{~d}(8.0)$ & $3.66 \mathrm{~d}(8.0)$ & $3.67 \mathrm{~d}(8.1)$ \\
\hline 8 & $1.94 \mathrm{~m}$ & $1.82 \mathrm{~m}$ & $1.80 \mathrm{~m}$ & $1.77 \mathrm{~m}$ \\
\hline 9 & $1.26 \mathrm{~m}$ & $1.02 \mathrm{~m}$ & $0.99 \mathrm{~m}$ & $1.02 \mathrm{~m}$ \\
\hline $11 \alpha$ & $1.76 \mathrm{~m}$ & $1.73 \mathrm{~m}$ & $1.74 \mathrm{~m}$ & $1.72 \mathrm{~m}$ \\
\hline $11 \beta$ & $1.58 \mathrm{~m}$ & $1.64 \mathrm{~m}$ & $1.60 \mathrm{~m}$ & $1.62 \mathrm{~m}$ \\
\hline $12 \alpha$ & $1.14 \mathrm{~m}$ & $1.18 \mathrm{~m}$ & $1.14 \mathrm{~m}$ & $1.14 \mathrm{~m}$ \\
\hline $12 \beta$ & $2.04 \mathrm{~m}$ & $2.03 \mathrm{~m}$ & $2.04 \mathrm{~m}$ & $2.08 \mathrm{~m}$ \\
\hline 14 & $1.37 \mathrm{~m}$ & $1.10 \mathrm{~m}$ & $1.06 \mathrm{~m}$ & $1.06 \mathrm{~m}$ \\
\hline $15 \alpha$ & $1.77 \mathrm{~m}$ & $1.67 \mathrm{~m}$ & $1.78 \mathrm{~m}$ & $1.37 \mathrm{~m}$ \\
\hline $15 \beta$ & $1.10 \mathrm{~m}$ & $1.30 \mathrm{~m}$ & $1.40 \mathrm{~m}$ & $1.18 \mathrm{~m}$ \\
\hline $16 \alpha$ & $1.88 \mathrm{~m}$ & $1.78 \mathrm{~m}$ & $1.67 \mathrm{~m}$ & $1.89 \mathrm{~m}$ \\
\hline $16 \beta$ & $1.30 \mathrm{~m}$ & $1.50 \mathrm{~m}$ & $1.28 \mathrm{~m}$ & $1.29 \mathrm{~m}$ \\
\hline 17 & $1.18 \mathrm{~m}$ & $1.21 \mathrm{~m}$ & $1.12 \mathrm{~m}$ & $1.10 \mathrm{~m}$ \\
\hline 18 & $0.77 \mathrm{~s}$ & $0.80 \mathrm{~s}$ & $0.78 \mathrm{~s}$ & $0.77 \mathrm{~s}$ \\
\hline 19 & $3.87 \mathrm{~d}(11.5), 3.58 \mathrm{~d}(11.5)$ & $3.86 \mathrm{~d}(11.7), 3.60 \mathrm{~d}(11.7)$ & $3.85 \mathrm{~d}(11.5), 3.60 \mathrm{~d}(11.5)$ & $3.86 \mathrm{~d}(11.6), 3.60 \mathrm{~d}(11.6)$ \\
\hline 20 & $1.45 \mathrm{~m}$ & $2.14 \mathrm{~m}$ & $2.05 \mathrm{~m}$ & $1.40 \mathrm{~m}$ \\
\hline 21 & $0.98 \mathrm{~d}(6.5)$ & $1.06 \mathrm{~d}(6.6)$ & $1.02 \mathrm{~d}(6.5)$ & $0.95 \mathrm{~d}(6.4)$ \\
\hline 22 & $1.57 \mathrm{~m}, 1.17 \mathrm{~m}$ & 5.58 dd $(15.8,8.8)$ & 5.22 dd $(15.0,8.0)$ & $1.02 \mathrm{~m}$ \\
\hline 23 & $2.09 \mathrm{~m}, 1.92 \mathrm{~m}$ & $5.93 \mathrm{~d}(15.8)$ & 5.30 ddd $(15.0,7.5,7.0)$ & $1.45 \mathrm{~m}, 1.81 \mathrm{~m}$ \\
\hline 24 & & & $1.82 \mathrm{dd}(13.0,6.5)$ & $1.29 \mathrm{~m}$ \\
\hline 25 & 2.23 heptet (6.5) & 2.55 heptet (6.6) & $1.57 \mathrm{~m}$ & $1.51 \mathrm{~m}$ \\
\hline 26 & $1.02 \mathrm{~d}(6.5)$ & $1.07 \mathrm{~d}(6.6)$ & $0.87 \mathrm{~d}(7.0)$ & $0.88 d(6.6)$ \\
\hline 27 & $1.03 \mathrm{~d}(6.5)$ & $1.06 \mathrm{~d}(6.6)$ & $0.87 \mathrm{~d}(7.0)$ & $0.88 \mathrm{~d}(6.6)$ \\
\hline 28 & $4.72 \mathrm{~s}, 4.65 \mathrm{~s}$ & $4.82 \mathrm{~s}, 4.84 \mathrm{~s}$ & & \\
\hline
\end{tabular}

a Assigned by COSY, HSQC, NOESY, and HMBC experiments.

b $J$ values (in $\mathrm{Hz}$ ) in parentheses.

NOESY correlations (Fig. 6) between $\mathrm{H}-8$ with all protons of $\mathrm{H}-7, \mathrm{H}-11 \beta, \mathrm{H}_{2}-19$, and $\mathrm{H}_{3}-18$ positioned the above protons on the same side of the molecule, and revealed the $\alpha$-orientation of the 7-OH. Compound 6 is thus 24-methylenecholest-5-en$3 \beta, 7 \alpha, 19$-triol 19-acetate.

Compound 7 possessed a molecular formula of $\mathrm{C}_{28} \mathrm{H}_{46} \mathrm{O}_{3}$ as derived from its HRESIMS. The spectral data of compound 7 (Tables 2 and 4) differed from compound 6 only in the 19hydroxyl functionality $\left[\delta_{\mathrm{H}} 3.87(1 \mathrm{H}, \mathrm{d}, J=11.5 \mathrm{~Hz})\right.$ and $3.58(1 \mathrm{H}$, $\left.\mathrm{d}, J=11.5 \mathrm{~Hz}) ; \delta_{\mathrm{C}} 63.6\left(\mathrm{CH}_{2}\right)\right]$.

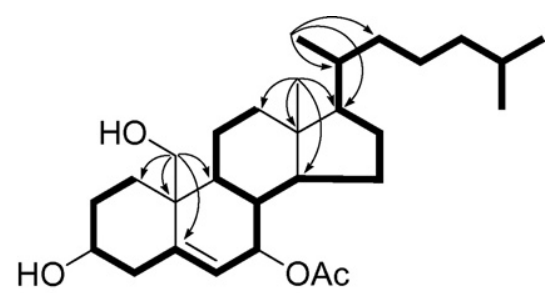

$$
\begin{aligned}
& \square:{ }^{1} \mathrm{H}-{ }^{1} \mathrm{H} \text { COSY correlations } \\
& \curvearrowright: \text { HMBC correlations }
\end{aligned}
$$

Fig. $4-{ }^{1} \mathrm{H}-{ }^{1} \mathrm{H}$ COSY and key HMBC correlations of 3.
The spectral data of 8 were quite similar to those of 9 isolated fron the black coral Antipathes subpinnata [19], except for NMR signals due to the side chain. As shown in Tables 2 and 4, signals due to a 22-E disubstituted double bond $\left[\delta_{\mathrm{H}} 5.22(1 \mathrm{H}, \mathrm{dd}\right.$, $J=15.0,8.0 \mathrm{~Hz})$ and $5.30(1 \mathrm{H}, \mathrm{ddd}, J=15.0,7.5,7.0 \mathrm{~Hz}) ; \delta_{\mathrm{C}} 139.7$ $(\mathrm{CH})$ and $127.6(\mathrm{CH})]$ appeared in the side chain of 9 , and those due to a $22 \mathrm{E}, 24(28)$-diene $\left[\delta_{\mathrm{H}} 5.58(1 \mathrm{H}, \mathrm{dd}, J=15.8,8.8 \mathrm{~Hz})\right.$ and $5.93(1 \mathrm{H}, \mathrm{d}, J=15.8 \mathrm{~Hz}) ; 4.82(1 \mathrm{H}, \mathrm{s})$ and $4.84(1 \mathrm{H}, \mathrm{s}) ; \delta_{\mathrm{C}} 135.8(\mathrm{CH})$, $129.2(\mathrm{CH}), 153.1(\mathrm{qC})$, and $\left.108.8\left(\mathrm{CH}_{2}\right)\right]$ were observed in $8 .{ }^{13} \mathrm{C}$ NMR spectral data of 9 and 10 were completely assigned, but ${ }^{1} \mathrm{H}$ spectral data were not completely assigned in the literature [19].

Compounds 8 and 10 exhibited cytotoxicity against P-388 with $\mathrm{ED}_{50}$ of 3.8 and $3.6 \mu \mathrm{g} / \mathrm{ml}$, respectively. Compounds 8 and

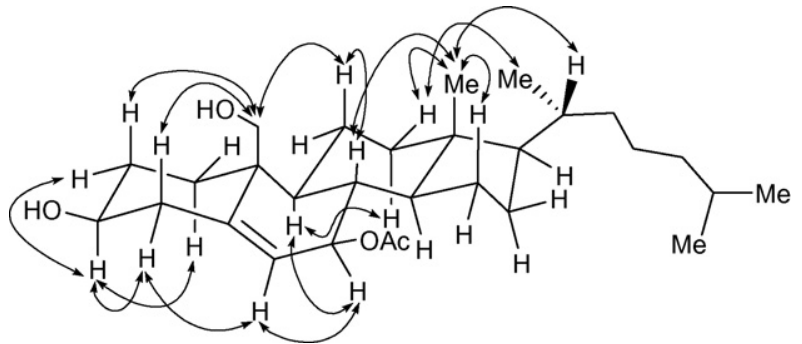

Fig. 5 - Selected NOESY correlations of 3. 


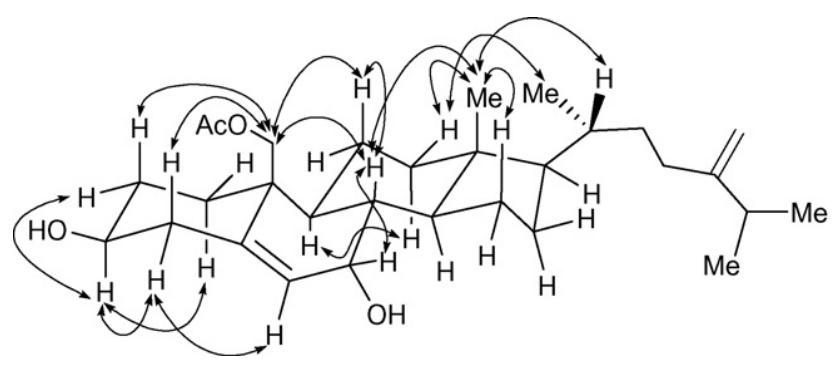

Fig. 6 - Selected NOESY correlations of 6.

10 exhibited cytotoxicity against HT-29 with $\mathrm{ED}_{50}$ of 4.7 and $4.3 \mu \mathrm{g} / \mathrm{ml}$, respectively. Nevertheless, compounds $1-6$ and 9 were not cytotoxic to P-388 and HT-29 cell lines.

\section{Acknowledgments}

Financial support was provided by Ministry of Education (C030313) and National Science Council of Taiwan (NSC 952323-B-110-003) awarded to C.Y. Duh.

\section{R E F E R E N C E S}

[1] Coll JC, Bowden BF, Tapiolas DM, Willis RH. Studies of Australian soft corals. XXXV. The terpenoid chemistry of soft corals and its implications. Tetrahedron 1985;41:1085-92.

[2] Poet SE, Ravi BN. 3 New diterpenes from a soft coral Nephthea species. Aust J Chem 1982;35:77-83.

[3] Ahond A, Bowden BF, Coll JC, Fourneron J, Mitchell SJ. Studies of Australian soft corals. 25. Several caryophyllene-based diterpenes from a Nephthea species. Aust J Chem 1981;34:2657-64.

[4] Blackman AJ, Bowden BF, Coll JC, Frick B, Mahendran M, Mitchell SJ. Studies of Australian soft corals. 29. Several new cembranoid diterpenes from Nephthea brassica and related diterpenes from a Sarcophyton species. Aust J Chem 1982;35:1873-80.

[5] Kitagawa I, Cui Z, Son BW, Kobayashi M, Kyogoku Y. Marine natural products.17. nephtheoxydiol, a new cytotoxic hydroperoxy-germacrane sesquiterpene, and related sesquiterpenoids from an Okinawan soft coral of Nephthea sp. (Nephtheidae). Chem Pharm Bull 1987;35:124-35.
[6] Bowden BF, Coll JC, Mitchell SJ. Studies of Australian soft corals. 21. A new sesquiterpene from Nephthea chabrolii and an investigation of the common clam Tridacna maxima. Aust J Chem 1980;33:1833-9.

[7] Handayani D, Edrada RA, Proksch P, Wray V, Witte L. New oxygenated sesquiterpenes from the Indonesian soft coral Nephthea chabrolii. J Nat Prod 1997;60:716-8.

[8] Duh CY, Wang SK, Weng YL, Brassicolene. a novel cytotoxic diterpenoid from the Formosan soft coral Nephthea brassica. Tetrahedron Lett 2000;41:1401-4.

[9] Duh CY, Wang SK, Weng YL, Chiang MY, Dai CF. Cytotoxic terpenoids from the Formosan soft coral Nephthea brassica. J Nat Prod 1999;62:1518-21.

[10] Rao MR, Venkatesham U, Venkateswarlu Y. 2 New 19-oxygenated polyhydroxy steroids from the soft coral Nephthea chabroli. J Nat Prod 1999;62:1584-5.

[11] Zhang WH, Williams ID, Che CT. Chabrolol-A, chabrolol-B and chabrolol-C, 3 new norditerpenes from the soft coral Nephthea chabroli. Tetrahedron Lett 2001;42:4681-6.

[12] Duh CY, Wang SK, Chu MJ, Sheu JH. Cytotoxic sterols from the soft coral Nephthea erecta. J Nat Prod 1998;61:1022-4.

[13] El-Gamal AAH, Wang SK, Dai CF, Duh CY. New nardosinanes and 19-oxygenated ergosterols from the soft coral Nephthea armata collected in Taiwan. J Nat Prod 2004;67:1455-8.

[14] El-Gamal AAH, Wang SK, Dai CF, Chen IC, Duh CY. Prenylbicyclogermacrane diterpenoids from the Formosan soft coral Nephthea pacifica. J Nat Prod 2005;68:74-7.

[15] Geran RI, Greenberg NH, MacDonald MM, Schumacher AM, Abbott BJ. Protocols for screening chemical agent and national products against animal tumors and other biological systems. Cancer Chemother Rep 1972;3:1-91.

[16] Hou RS, Duh CY, Chiang MY, Lin CN, Sinugibberol. a new cytotoxic cembranoid diterpene from the soft coral Sinularia gibberosa. J Nat Prod 1995;58:1126-30.

[17] Bortolotto M, Braekman JC, Daloze D, Tursch B. Chemical studies of marine invertebrates. XXIX. $4 \alpha$-Methyl-3 $\beta, 8 \beta$-dihydroxy-5 $\alpha$-ergost-24(28)-en-23-one, a novel polyoxygenated sterol from the soft coral Litophyton viridis (coelenterate, octocorallia, alcyonacea). Steroids 1977;30:159-64.

[18] Bortolotto M, Braekman JC, Daloze D, Losman D, Tursch B. Chemical studies of marine invertebrates. XXIII. A novel polyoxygenated sterol from the soft coral Litophyton viridis (coelenterate, octocorallia, alcyonacea). Steroids 1976;28:461-6.

[19] Aiello A, Fattorusso E, Menna M. Four new bioactive polyhydroxylated sterols from the black coral Antipathes subpinnata. J Nat Prod 1992;55:321-5. 